\title{
Search for DHEASism and DHEASoma
}

Tomas Kurakovas ${ }^{1}$, Ilona Banisauskaite ${ }^{1}$, Birute Zilaitiene ${ }^{1}$, Rytas Ostrauskas ${ }^{1}$ Vaidotas Urbanavicius ${ }^{2}$, Valentinas Matulevicius ${ }^{1}$, Rasa Verkauskiene ${ }^{1}$, Irina Bilodid ${ }^{3}$

1 Institute of Endocrinology, Lithuanian University of Health Sciences, Kaunas, Lithuania,

${ }^{2}$ Faculty of medicine, Vilnius University, Vilnius, Lithuania, ${ }^{3}$ University of medicine, Minsk, Belarus

\section{Introduction}

Recently we described the clinical case

36-year-old woman was referred because of the secondary amenorr hirsutism, weight gain, decreased sexuality and epileptic seizures.

On CT tumor in the right adrenal region was discovered

High increase of DHEAS and testosterone was observed.

The symptoms of the disease disappeared immediately after surgery and last until now (24 months)

- The goal of the study was to find more adrenal DHEAS secreting tumors cases.

\section{Methods}

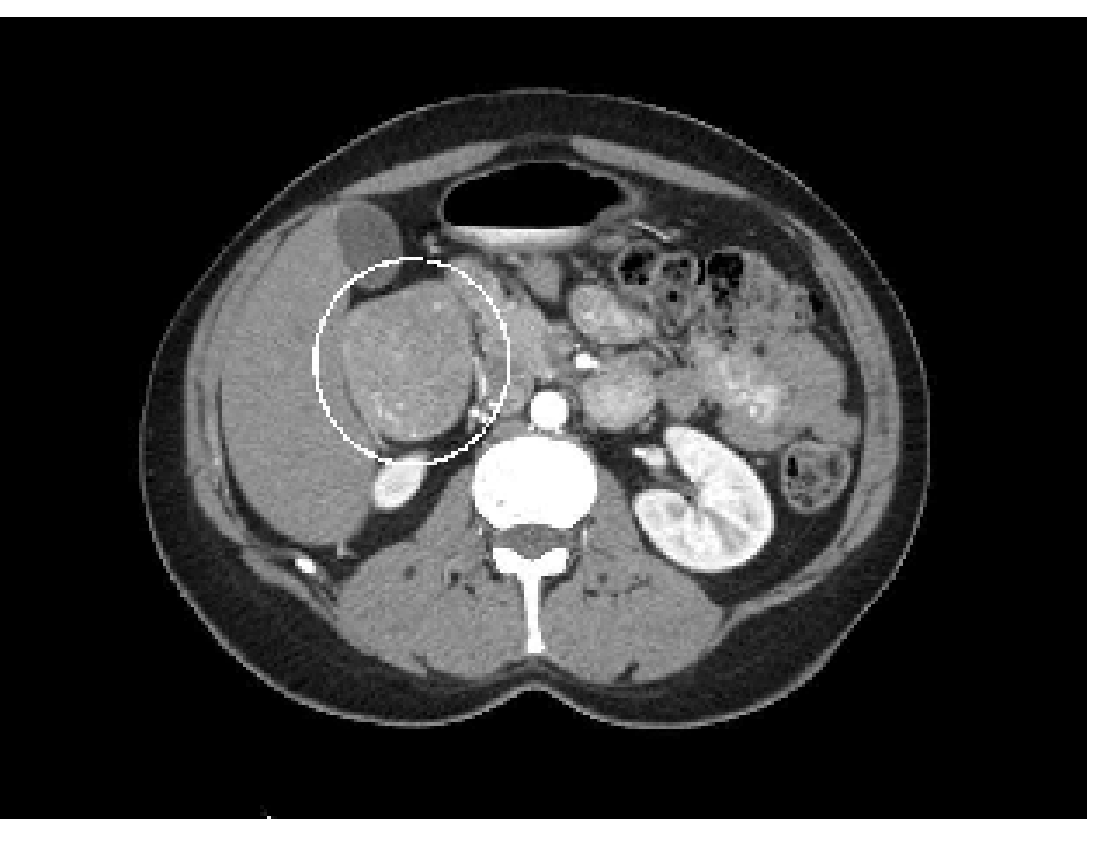

\section{Conclusion}

DHEAS-centered investigation of 1215 women, referred for DHEAS investigation because of the hirsutism, menstrual cycle disorders or infertility, revealed that the dehydroepiandrosteronism (DHEASism) is found frequently - in 11, $8 \%$. On contrast to that, the dehydroepiandrosteroma (DHEASoma) was found rarely - only in 3 patients. More prospective investigations using not only the determination of DHEAS, but also the clinical findings would be performed for detection of DHEASoma.

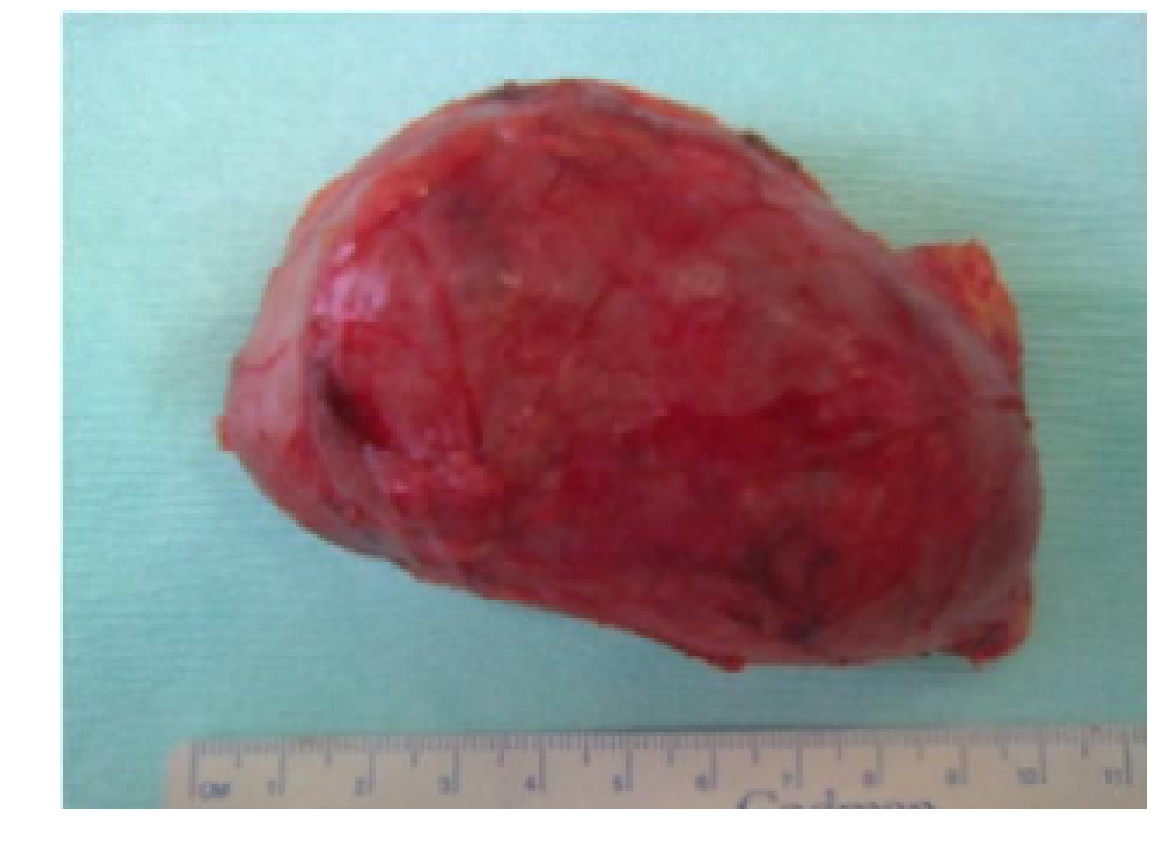

DHEAS-centered investigation for DHEASism / DHEASoma.

Search of increased DHEAS according to the lists of laboratory investigations at Kaunas and Vilnius university hospitals during 1 year -2014

Women were referred for investigation of DHEAS because of hirsutism, menstrual circle disturbances and infertility.

Search for medical records with high DHEAS.

Search of clinical, laboratory and imaginative data from medical records.

\section{Results}

Increased DHEAS was considered, when the patient's DHEAS concentration was higher than the maximal value of DHEAS for age, indicated in the assay kit.

Ratio $\frac{\text { DHEAS patient }}{\text { DHEAS maximal value }}>1$ was found in $11.76 \%$ of women referred for the investigation Mild increase was in $87.4 \%\left(\frac{R}{\text { DHEAS }}<1,3\right)$, moderate increase was in $9.8 \%\left(1.3<\frac{R}{\text { DHEAS }}<2\right)$, high increased was in $2.8 \%\left(\frac{\mathrm{R}}{\text { DHEAS }}>2\right)$

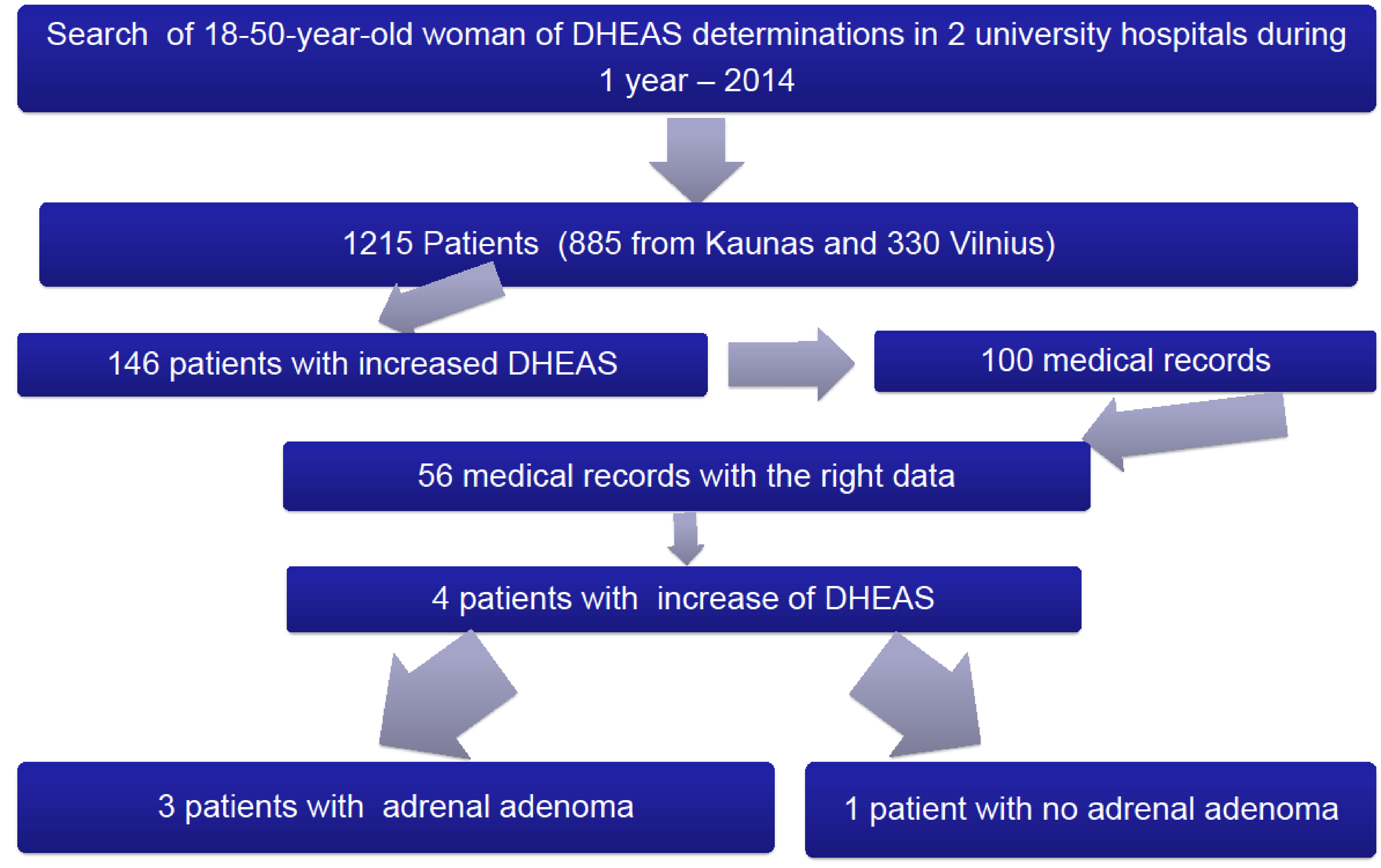

We managed to analyze outpatient files of 56 patients, that have R/ DHEAS >1.

Results of age, height, weight, BMI and DHEAS in the whole group are shown in the table 1

\begin{tabular}{|c|c|c|c|c|c|}
\hline \multicolumn{7}{|c|}{ R/DHEAS >1 } \\
\hline & Age & Height & Weight & BMI & R/DHEAS \\
\hline Mean & 26,32 & 168,55 & 80,38 & 27,87 & 1,22 \\
\hline Standard Deviation (SD) & 6,12 & 5,76 & 21,08 & 7,18 & 0,24 \\
\hline Count (n) & 56 & 56 & 56 & 56 & 56 \\
\hline
\end{tabular}

Compared the results of patients with R/DHEAS $<1,3$ and R/DHEAS $>1,3$

\begin{tabular}{|c|c|c|c|c|c|c|c|c|c|c|}
\hline & \multicolumn{2}{|c|}{ Age } & \multicolumn{2}{c|}{ Height } & \multicolumn{2}{c|}{ Weight } & \multicolumn{2}{|c|}{ BMI } & \multicolumn{2}{|c|}{ R/DHEAS } \\
\hline Mean & 27,1 & 24,37 & 169,16 & 167,03 & 81,70 & 77,09 & 28,29 & 26,82 & $\begin{array}{c}1,1 \\
0\end{array}$ & 1,52 \\
\hline $\begin{array}{c}\text { Standard } \\
\text { derivation } \\
\text { (SD) }\end{array}$ & 6,59 & 4,30 & 5,98 & 5,02 & 21,71 & 19,70 & 7,45 & 6,57 & 0,11 & 0,21 \\
\hline Count (n) & 40 & 16 & 40 & 16 & 40 & 16 & 40 & 16 & 40 & 16 \\
\hline
\end{tabular}

Table 2. Phenotypic data of patients with R/DHEAS $<1,3$ and R/DHEAS $>1,3$

R/DHEAS $<1,3$

R/DHEAS $>1,3$

3 patients with increased DHEAS and adrenal adenoma.

\begin{tabular}{|c|c|c|c|c|c|c|c|}
\hline $\begin{array}{c}\text { Patient } \\
\text { No. }\end{array}$ & Symptoms & Age & Weight & High & BMI & R/DHEAS & CT \\
\hline 2.(J.P.) & $\begin{array}{c}\text { Hirsutism, } \\
\text { weight gain }\end{array}$ & 33 & 98 & 173 & 32 & 1,435 & $\begin{array}{c}\text { Tumor in the } \\
\text { left adrenal } \\
\text { region }\end{array}$ \\
\hline $\begin{array}{c}\text { Hirsutism, } \\
\text { secondary } \\
\text { amenorrhea }\end{array}$ & 27 & 65 & 172 & 21,5 & 1,3 & $\begin{array}{c}\text { Left adrenal } \\
\text { hyperplasia }\end{array}$ \\
\hline 3.(J.R.) & $\begin{array}{c}\text { Hirsutism, } \\
\text { acnea }\end{array}$ & 31 & 68 & 165 & 24,97 & 1,46 & $\begin{array}{c}\text { Tumor in the } \\
\text { left adrenal } \\
\text { region }\end{array}$ \\
\hline
\end{tabular}

Table 3. Phenotypic data of patients with adrenal adenoma

Case report about one patient with increased DHEAS, but without adrenal tumor - 37 year-old woman referred because of inability to get pregnant, primary amenorrhea; - Obj.: High-152 cm, Weight -78 kg, BMl- 34,2 kg/m2, short neck, small breasts, multiple pigmented moles, large ears.
Hormones
-17-OH-P - 2,82nmol/
- DHEA-S - 26,8 umol// $\quad$ Prolactin - $130 \mathrm{mU} / \mathrm{l}$
- Testosterone- 6,05nmol/l $\bullet$ FT4 - 10,52 pmol//;
- SHBG - $23 \mathrm{nmol} / \mathrm{l}(\mathrm{LAl} \cdot \mathrm{TSH}-4,63 \mathrm{mU} / \mathrm{l}$;
26,3)
- FSH- 29,4 U/
- Anti-TPO - 948,08 k
- Cortisol - $386 \mathrm{nmol} / \mathrm{l}$
Progesterone- 6,2 nmol/l
Karyotype blood test showed the typical Turne syndrome with $70 \%$ of $45 X 0$ and the rest is the mosaicism with $Y$ chromosome.
$\underline{\mathrm{CT}}$ : Both adrenal glands without additional derivative.

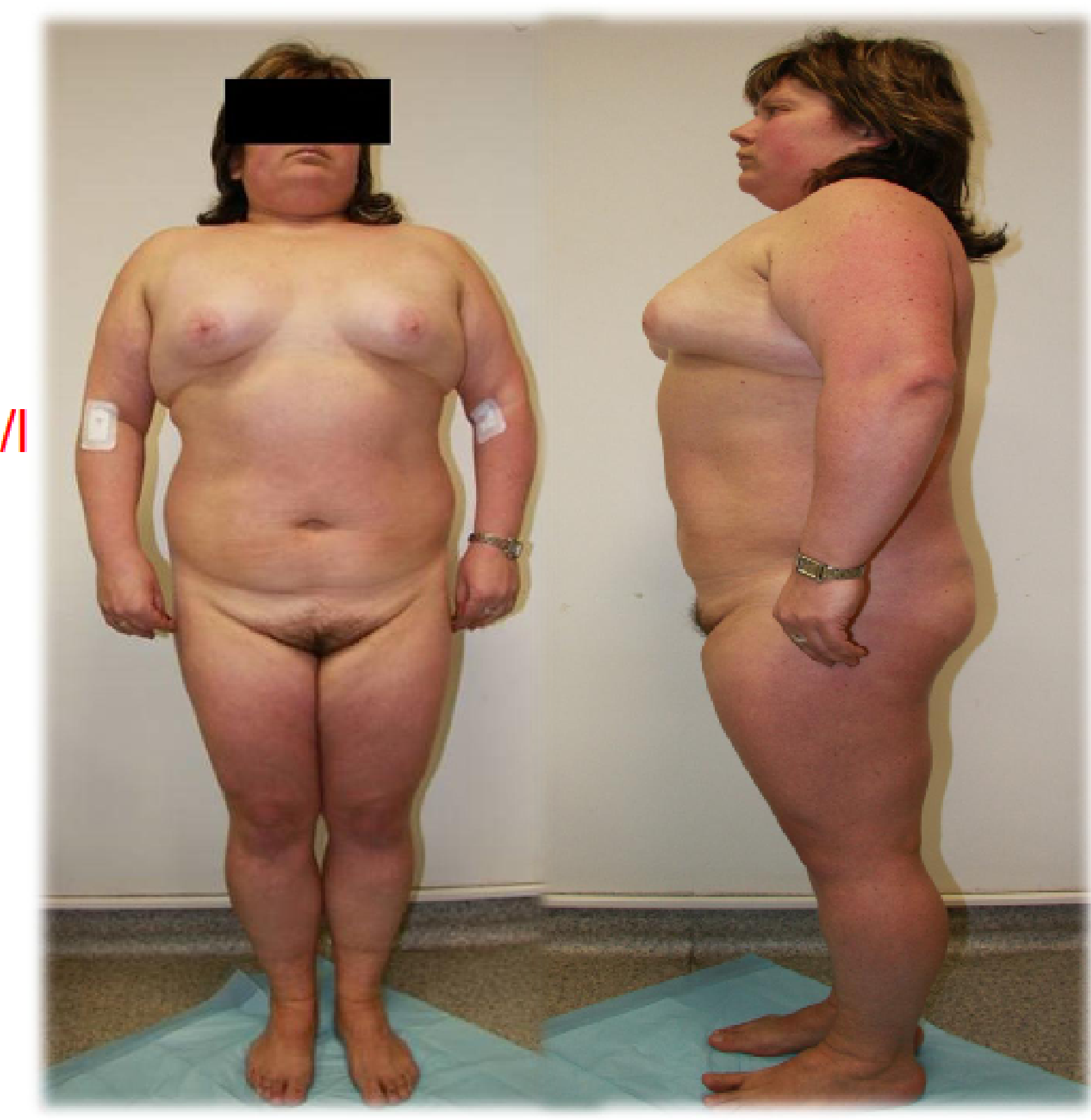

Gynecologist consultation

Labia minora are visualized, but the labia majora are absent. Clitoris is enlarged and the uterus is small.

US: uterus 2,89 x 1,40 x 4,70 cm; Thread-like endometrium. Right ovary - 0,8 $\times 0,5 \mathrm{~cm}$. Left ovary - not visualized

Diagnosis: Turner syndrome; Ovary and labia majora dysgenesis; Primary amenorrhea; Hypoplastic uterus; idiopathic DHEASism; Autoimmune thyroiditis; Eutirosis.

\section{Discussion}

The DHEASism is diagnosed often, especially when polycystic ovary is diagnosed $2,3,4$ When search for DHEASism/DHEASoma is centered on DHEAS investigation, during one year we were able to suspect 3 patients with DHEASoma, which is a rare disease $e^{5,6,7}$

Retrospective analyses are not so informative, due to the fact that the medical records are frequently very bad documented. Prospective analyses should be done in multinational and multicentral studies. 